\title{
Narrative Review: Botulinum Toxin Applications in Sports Medicine
}

\author{
Kuwabara $\mathrm{A}^{1 *}$ and Fredericson $\mathrm{M}^{2}$ \\ ${ }^{1}$ Resident Physician, Department of Physical Medicine and Rehabilitation, Stanford University, USA \\ ${ }^{2}$ Professor of Orthopaedic Surgery, Department of Physical Medicine and Rehabilitation, Stanford University Medical Center, USA \\ *Corresponding author: Resident Physician, Stanford University, Department of Physical Medicine and Rehabilitation, Bradford \\ Street, Redwood City, USA
}

\begin{abstract}
Due to the botulinum toxin's (BoNT) direct and highly selective ability to induce hypotonia as well as its potential to confer potent antinociceptive efficacy, we hypothesize that there may be potential applications in sports medicine populations, which can improve pain, range of motion, and ultimately return to play. From review of the literature, we have identified four different areas of neuromuscular dysfunction for which treatment with BoNT has been shown to be effective: overactivity syndromes, muscular imbalances, bio protection, and neuromuscular pain. BoNT has emerging evidence of multiple beneficial applications in sports medicine. The evidence is strongest for plantar fasciitis and hip osteoarthritis. It is an effective and transient therapeutic option that may improve return to play times.
\end{abstract}

\section{Introduction}

Intramuscular injections of botulinum toxin (BoNT) induce muscle relaxation by blocking acetylcholinesterase at the neuromuscular junction by cleavage of synaptosomal associated protein $25 \mathrm{kDa}$ (SNAP-25) [1]. There are 7 serotypes (A-G) of which only $A$ and $B$ are used clinically. There are four different FDA-approved therapeutic BoNTs (Onabotulinumtoxin A (BoNT-A), Abobotulinumtoxin A (AboBoNT-A), Incobotulinumtoxin A, and Rimabotulinumtoxin B (BoNT-B)). FDA-approved clinical indications include blepharospasm, cervical dystonia, chronic migraine, focal spasticity, overactive bladder, severe axillary hyperhidrosis, strabismus, and cosmetic applications (e.g. glabellar lines or wrinkles). Side effects include pain or irritation at the injection site, respiratory depression, and dysphagia. Contraindications include hypersensitivity to any preparation or components and active infection at proposed injection site. There are no known drug interactions. The onset of its effect occurs over days to weeks and generally lasts for about 3 months. Due to the toxin's direct and highly selective ability to induce hypotonia as well as its potential to confer potent antinociceptive efficacy, we hypothesize that there may be potential off-label applications in sports medicine populations, which can improve pain, range of motion, and ultimately return to play. From review of the literature, we have identified four different areas of neuromuscular dysfunction for which treatment with BoNT has been shown to be effective: overactivity syndromes, muscular imbalances, bio protection, and neuromuscular pain.

\section{Method}

A search was conducted April 1, 2019. References for this narrative review were obtained using a search of online databases PubMed, Medline, Cochrane, and CINAHL were used to search for articles published using the terms botulinum toxin OR Botox, chemodenervation, and sports OR sports medicine. Some of the references were not identified but were obtained through reference lists of other articles. Our inclusion criteria were studies that looked primarily at sports medicine specific applications of BoNT. Eligibility of studies was assessed by 2 reviewers. BoNT is used to treat a wide variety of conditions. For clarity, we have grouped indications into the following subsections.

\section{Overactivity syndromes}

\section{A Plantar fasciitis}

Background: Plantar fasciitis is the most common cause of foot pain, affecting $10 \%$ of the US population [2,3]. Driven by the combination of repetitive opposing traction by the Achilles tendon and the forefoot windlass effect, microscopic tears occur in the central bundle of the plantar fascia [4,5]. BoNT-A injection of the calf muscles and plantar has shown some clinical promise in 
easing contractures and improving symptoms in short-term studies described below. Tsikopoulos [6] compared different injection therapies for plantar fasciitis in a review of 22 trials. For pain relief, BoNT-A provided a significant short-term advantage over placebo up to 6 months. Dehydrated amniotic membrane injections were significantly superior to corticosteroids in the short-term but lacked data beyond 2 months. Recent studies have demonstrated that platelet-rich plasma (PRP) provided longer-term relief (1218 months) for plantar fasciitis compared to corticosteroids. However, there have been no direct comparison studies of BoNT-A to amniotic membrane or PRP [7,8]. Elizondo-Rodriguez [9] performed a randomized control trial of 36 patients that compared intramuscularly applied BoNT-A in the Gastric-soleus complex with intralesional steroids for plantar fasciitis. When compared to patients who received steroids, the patients who received BoNT-A exhibited more rapid and sustained improvement over 6 months. Babcock [10] performed a randomized control trial of 27 patients (43 feet) with plantar fasciitis. Compared with placebo, the BoNT-A group significantly improved in all measures at both 2 and 8 weeks after treatment. No side effects were noted (Table1).

Table 1: Summary of recommendations based on current literature.

\begin{tabular}{|c|c|}
\hline Pathology & Recommendation \\
\hline Plantar Fasciitis & A \\
\hline Chronic Exertional Compartment Syndrome & C \\
\hline Functional Popliteal Artery Entrapment Syndrome & C \\
\hline Lateral epicondylitis & B \\
\hline Accessory Soleus Muscle & C \\
\hline Runner's dystonia & C \\
\hline Muscular imbalance causing knee pain & C \\
\hline Piriformis Syndrome & B \\
\hline Shoulder impingement & B \\
\hline Rotator Cuff Repair Immobilization & B \\
\hline Increasing Dorsiflexion after Achilles Tendon \\
Repair & C \\
\hline Osteoarthritis: Hip/Knee & I \\
\hline Whiplash Associated Disorder & I \\
\hline
\end{tabular}

Conclusion grade A recommendation: There is level I evidence that BoNT can improve pain and function in patients with plantar fasciitis.

\section{B Chronic exertional compartment syndrome (CECS)}

Background: Chronic exertional compartment syndrome is characterized by compartment pain triggered by exercise and abnormally high compartment pressure after exercise. The pathophysiology is due to discrepancy between muscle size and its constricting fascial compartment, muscle ischemia, or stimulation of local nociceptors. Given a proposed role of muscle hypertrophy, BoNT-A could help in the management of CECS by reducing the volume of the compartment content. Baria [11] presented a case with continued pain relief after AboBoNT-A and had resumed her active lifestyle without any adverse effects up to 14 months. In a case series by Isner-Horobeti [12], AboBoNT-A was injected into the muscles of 16 moderately trained patients diagnosed with anterior or anterolateral exertional compartment syndrome of the leg (25 anterior compartments and 17 lateral compartments). At 9-month follow, the exertional pain was eliminated in 15 patients. In 5 patients, the strength of the injected muscles remained normal. In 11 patients, strength decreased significantly from 4.5 (1 month after the first injection) to 3.5 (at final follow up), although without functional consequences.

- Conclusion-grade $\mathbf{C}$ recommendation: There is level IV-V evidence that BoNT can improve pain in patients with CECS without functional strength loss up to 9-14 months.

\section{Functional popliteal artery entrapment syndrome}

Background: Functional popliteal artery entrapment syndrome (FPAES) is notable cause of exercise-induced muscle leg pain that is often due to the increased bulk of the gastrocnemius muscles [13]. proposed mechanisms of action for intramuscular periarterial BoNT therapy for FPAES are [14] 1) paralysis and/or localized atrophy of the muscle responsible for the dynamic arterial occlusion, and 2) possible arterial smooth muscle relaxation of the popliteal artery resulting in vasodilatation. Murphy [15] published a case report regarding an elite male athlete with FPAES. The athlete experienced a decrease in strength and performance at 1-week post-injection but returned to baseline within four weeks. The athlete had an increase in his sprint distance per game post-injection. On ultrasound, he had visible medial gastrocnemius denervation atrophy with a decrease in arterial flow velocity at four weeks post-injection. Hislop [16] published a case series of 27 patients that received ultrasound-guided BoNT-A injection at the level of artery occlusion. No patients reported being worse off after the intervention; 59\% of patients had a good response (initial improvement maintained at 12 months), $22 \%$ a mixed response (initial improvement that reduced over 12 months), and $19 \%$ a poor response (no difference) to treatment. Isner-Horobeti [17] published a case of a soldier with bilateral functional popliteal artery entrapment syndrome that did not improve after bilateral popliteal arteriolysis without resection of the gastrocnemius medial head. After AboBoNT-A, follow-up (from 1 month to 3 years after BoNT treatment) showed disappearance of exercise-induced pain and improvement of the physical and sports performance.

Conclusion - grade C recommendation: There is level IV-V evidence BoNT can improve symptoms of FPAES however, athletes may experience a transient decrease in function.

\section{Lateral epicondylitis}

- Background: lateral epicondylitis refers to pain in the lateral elbow due to repetitive wrist Oskarsson [18] demonstrated low intramuscular blood flow in the extensor carpi radialis brevis (ECRB) in patients with lateral epicondylitis, postulating that mechanism of injury is due to decreased microcirculation from persistent muscle contraction. Anaerobic metabolism would subsequently cause lactate accumulation and muscle swelling. 
Therefore, muscle relaxation induced by BoNT may improve microcirculation and swelling. Creuze [19] performed a randomized control study of 60 patients with chronic lateral epicondylitis resistant to treatment for $>6$ months. Pain intensity and the effect on quality of life were both significantly lower in the group treated with AboBoNT-A compared with placebo at day 90. The rate of clinically detected transitory paresis of the third finger on extension was $17.2 \%$ in the AboBoNT-A group without functional impairment. Lian [20] performed a systematic review of 36 randomized control trials. BoNT improved pain at mid-term follow up. All treatments increased adverse events compared with placebo. $92 \%$ of patients experienced pain resolution after receiving placebo within 4 weeks of follow-up. Dong's [21] systematic review demonstrated a significant difference between BoNT and placebo by the pairwise meta-analysis results but became non-significant during network meta-analysis. Krogh [22] performed a systematic review of 17 trials to assess the comparative effectiveness and safety of injection therapies in patients with lateral epicondylitis. Although BoNT showed marginal benefit, it caused temporary paresis of finger extension, and all trials were at high risk of bias. Oskarsson [23] published a case series of 10 patients with unilateral epicondylitis and decreased intramuscular blood flow in ECRB. The difference in intramuscular blood flow between the control and the affected side had decreased 3 and 12 months after AboBoNT-A. Lactate concentration at the 12-month follow-up had decreased while perceived pain was reduced and function in daily activities had improved.

- Conclusion - Grade B Recommendation: There is level I-IV evidence that BoNT-A can improve pain in lateral epicondylitis, however, care must be taken to avoid paresis of the third finger.

\section{E Accessory soleus muscle}

- Background: Symptomatic accessory soleus muscle (ASM) can cause exercise-induced leg pain due to local nerve/ vascular compression, muscle spasm, or local compartment syndrome. As intramuscular injections of BoNT-A can reduce muscle tone and mass, it may alleviate symptomatic ASM. IsnerHorobeti [24] published a case series of 5 patients. After BoNT-A injection, exertional pain disappeared, and all five patients resumed their normal level of physical and sports performances. Neither side effects nor motor deficits were observed.

- Conclusion - Grade C Recommendation: There is level IV evidence that BoNT-A is well tolerated and can improve pain in patients with ASM for years after treatment.

\section{F Task-specific dystonia: runner's dystonia}

- Background: Task-specific dystonias seen in sports medicine include Runner's dystonia and golfer's yips [25]. Taskspecific movement disorders constitute a group of hyperkinetic movement disorders that occur only during certain activities, postures, or circumstances such as a writer's cramp. BoNT has provided symptom relief through relaxation of the affected muscle. The retrospective case series by Ahmad [26] presented surface electromyography (EMG) and joint kinematic data from 13 patients with RD who underwent instrumented gait analysis
(IGA) for muscle selection for BoNT therapy. Nine patients received BoNT therapy based on the results of IGA, and 7/9 reported some benefit. Three patients reported significant benefit allowing them to continue long-distance running. The remaining four patients reported variable levels of benefit, allowing some to continue training at lower intensity levels while others reported only a shortlived treatment response. Two patients had no response to BoNT at high doses. The retrospective review of 20 patients with runner's dystonia by Cutsforth-Gregory [27] found that BoNT, levodopa, clonazepam, trihexyphenidyl, and physical therapy provided modest benefit to some, but all patients remained substantially symptomatic at last follow up (up to 4.2 years after diagnosis). The case series of 5 patients by $\mathrm{Wu}$ [28] examined the effects of BoNT on the dystonia of 1 leg during long-distance running. One patient benefited from an oral anticholinergic, 1 from levodopa, and 2 patients benefited from repeat BoNT injections.

- $\quad$ Conclusion - Grade C Recommendation: There is level III-IV evidence that BoNT-A can improve symptoms in patients with runner's dystonia.

\section{Muscular imbalances}

\section{A. Anterior knee pain}

- Background: Muscle overactivity in neurologically normal muscle, where an imbalance exists between a relatively overactive muscle and its less active synergist or antagonist, can inhibit control of the antagonist producing a functional muscle imbalance [29]. Intramuscular injection of BoNT has the potential to correct imbalances by the reduction of focal muscle overactivity and localized muscle spasm. The case series of 45 patients by Stephen [30] investigated the effect of an ultrasound-guided AboBoNT-A injection into the tensor fasciae latae, followed by physical therapy, in patients classified with lateral patellofemoral overload syndrome who failed to respond to conventional treatment. There was a significant improvement in Anterior Knee Pain Scale (AKPS) scores from before the injection to 1, 4, and 12 weeks after the injection and in $87 \%$ of patients at approximately 5 years after the injection. A significant effect on the modified Ober test was identified as a result of the intervention, with an increase in leg drop found at 1, 4, and 12 weeks after the injection compared with before the injection.

The randomized controlled crossover trial by Singer [31] examined the efficacy of AboBoNT-A injection plus an exercise program to remediate chronic anterior knee pain associated with quadriceps muscle imbalance in 24 patients. The injection targeted the vastus lateralis with the intention of disinhibiting the vastus medialis. Improvement at 12 weeks was significantly greater for BoNT-A compared with placebo-injected subjects for the AKPS, pain on kneeling, squatting and level walking. At 24 weeks, 16 of 19 BoNT-A-injected and 2 of the remaining 5 placebo-injected subjects were either satisfied or very satisfied with treatment outcomes. Improvements were maintained in 11 of 14 BoNT-A-injected and 2 of 5 placebo subjects available at longer-term follow-up. A follow up study demonstrated that improvements were sustained at follow-up, with an average benefit of 34 months post-injection in 
44/57 cases. Cullen [32] presented two cases with improved pain functional muscle balance. After two weeks, one case was able to produce an isolated gluteal muscle contraction after receiving 500 units of AboBoNT-A in her left upper hamstrings. The second case had relief after two injection episodes of AboBoNT-A into the distal vastus lateralis and tensor fascia latae.

- Conclusion - grade $\mathrm{C}$ recommendation: There is level IV evidence that intramuscular injection of BoNT, in carefully selected cases, provides short term reduction of focal muscle overactivity, and may facilitate activation of relatively 'inhibited' muscles and assist the restoration of more appropriate motor patterns (Table 2-a-c)

Table 2: Summary of evidence based on current literature (new to old). U = units of Onabotulinum toxin-type A unless specified otherwise.

\begin{tabular}{|c|c|c|c|c|c|}
\hline \multicolumn{6}{|c|}{ Nociception } \\
\hline Pathology & Author & Design & Protocol & Results & Evidence \\
\hline \multirow[t]{4}{*}{ Osteoarthritis } & Eleopra [41] & $\begin{array}{l}\text { Randomized, Double- } \\
\text { Blinded, Placebo- } \\
\text { Control }\end{array}$ & $\begin{array}{l}\text { 400U AboBoNT-A into } \\
\text { adductor muscles }\end{array}$ & $\begin{array}{c}\text { 4-week Harris Hip Score \& VAS significant } \\
\text { improvements }\end{array}$ & I \\
\hline & Hassan [42] & $\begin{array}{l}\text { Systematic review, } \\
\text { nonhomogenous }\end{array}$ & & Limited supporting evidence & II \\
\hline & \multirow[t]{2}{*}{ Mahowald 2016} & \multirow[t]{2}{*}{ Case series } & \multirow[t]{2}{*}{$\begin{array}{c}25-50 \mathrm{U} \text { in } 3 \text { knees and } \\
3 \text { ankles and } 450-100 \\
\text { units in } 9 \text { shoulders }\end{array}$} & $\begin{array}{c}\text { Significant } 55 \% \text { decrease in lower } \\
\text { extremity pain, } 36 \% \text { improvement in } \\
\text { Timed Stands Test, } 71 \% \text { reduction in } \\
\text { shoulder pain, active range of motion } \\
\text { increased in flexion } 67 \% \text { and abduction } \\
42 \%\end{array}$ & \multirow[t]{2}{*}{ IV } \\
\hline & & & & Duration of pain relief 3-12 months & \\
\hline \multirow[t]{5}{*}{ Whiplash } & Teasel [44] & $\begin{array}{l}\text { Systematic Review - } \\
\text { nonhomogenous }\end{array}$ & 3 RCTs \& 1 case series & $\begin{array}{l}\text { Contradictory evidence during chronic } \\
\text { stage (>12 weeks after injury) }\end{array}$ & II \\
\hline & Braker [45] & $\begin{array}{l}\text { Randomized, Double- } \\
\text { Blinded, Placebo- } \\
\text { Control }\end{array}$ & $\begin{array}{l}20 \text { patients with } \\
\text { cervical myofascial } \\
\text { pain } 2-48 \text { weeks after } \\
\text { whiplash who had } \\
200 \mathrm{U} \text { into } 4 \text { tender } \\
\text { points }\end{array}$ & $\begin{array}{l}\text { Greater percentage of patients who } \\
\text { received BoNT achieved a } 50 \% \text { reduction } \\
\text { in pain intensity at } 24 \text { weeks but more } \\
\text { adverse effects }\end{array}$ & I \\
\hline & Padberg [46] & $\begin{array}{l}\text { Randomized, Double- } \\
\text { Blinded, Placebo- } \\
\text { Control }\end{array}$ & $\begin{array}{l}40 \text { patients with grade } \\
1-2 \text { whiplash received } \\
100 \mathrm{U} \text { into muscles } \\
\text { with increased } \\
\text { tenderness }\end{array}$ & At 12 weeks, no significant difference & I \\
\hline & Juan [47] & $\begin{array}{l}\text { Randomized, Double- } \\
\text { Blinded, Placebo- } \\
\text { Control }\end{array}$ & $\begin{array}{l}5 \text { injections of } 20 U \text { into } \\
1 \text { or more of: splenius } \\
\text { capitis, semispinalis } \\
\text { capitis, trapezius }\end{array}$ & No final data & I \\
\hline & Freund 2002 & $\begin{array}{l}\text { Randomized, Double- } \\
\text { Blinded, Placebo- } \\
\text { Control }\end{array}$ & $\begin{array}{l}28 \text { patients with } \\
\text { chronic grade } 2 \text { WAD } \\
\text { 100U AboBoNT-A or } \\
\text { saline in splenius, } \\
\text { capitis, rectus }\end{array}$ & Significant reduction in pain at 4 weeks & I \\
\hline
\end{tabular}

Table 2a.

\begin{tabular}{|c|c|c|c|c|c|}
\hline \multicolumn{6}{|c|}{ Overactivity Syndromes } \\
\hline Pathology & Author & Design & Protocol & Results & Evidence \\
\hline \multirow[t]{3}{*}{ Plantar Fasciitis } & Tsikopoulos [6] & $\begin{array}{l}\text { Systematic review \& } \\
\text { network meta-analysis } \\
\text { of } 22 \text { randomized } \\
\text { controlled trials }\end{array}$ & & $\begin{array}{l}\text { Provided significant short- } \\
\text { term advantage over placebo } \\
\text { that was still present at } 6 \\
\text { months }\end{array}$ & I \\
\hline & $\begin{array}{l}\text { Elizondo- } \\
\text { Rodriguez [9] }\end{array}$ & $\begin{array}{l}\text { Randomized, Double- } \\
\text { Blinded, Placebo- } \\
\text { Control }\end{array}$ & $\begin{array}{l}\text { 100U each medial \& lateral } \\
\text { gastrocnemius \& } 50 U \text { soleus }\end{array}$ & $\begin{array}{c}\text { Rapid \& sustained } \\
\text { improvement compared to } \\
\text { steroids over } 6 \text { months }\end{array}$ & I \\
\hline & Babcock [10] & $\begin{array}{l}\text { Randomized, Double- } \\
\text { Blinded, Placebo- } \\
\text { Control }\end{array}$ & $70 \mathrm{U}$ divided into 2 sites per foot & $\begin{array}{l}\text { Improved pain and function } \\
\text { at 3- and 8-week follow up }\end{array}$ & I \\
\hline
\end{tabular}




\begin{tabular}{|c|c|c|c|c|c|}
\hline \multirow{4}{*}{$\begin{array}{l}\text { Chronic exertional } \\
\text { compartment } \\
\text { syndrome (CECS) }\end{array}$} & \multirow{4}{*}{ Baria [11] } & \multirow{4}{*}{ Case report } & \multirow{4}{*}{$\begin{array}{l}\text { 20U AboBoNT-A proximally \& } \\
\text { 20U distally in the following } \\
\text { on the left leg: Tibialis anterior, } \\
\text { extensor hallucis longus, } \\
\text { extensor digitorum longus, } \\
\text { fibularis longus, fibularis brevis }\end{array}$} & $\begin{array}{l}\text { Pain decreased to } 1 / 10 \text { from } \\
9 / 10 \text { in } 1 \text { week }\end{array}$ & \multirow{4}{*}{$\mathrm{V}$} \\
\hline & & & & $\begin{array}{l}\text { jogging short distances } \mathrm{w} / \mathrm{in} \\
2 \text { weeks }\end{array}$ & \\
\hline & & & & $\begin{array}{l}1 \text { month increased to } 2-3 \\
\text { miles several times/weeks }\end{array}$ & \\
\hline & & & & $\begin{array}{l}14 \text { months: no further pain, } \\
\text { symmetric leg circumference } \\
\text { \& normal strength }\end{array}$ & \\
\hline & $\begin{array}{c}\text { Isner-Horobeti } \\
{[12]}\end{array}$ & Case series & $\begin{array}{c}25 \text { anterior compartments \& } 17 \\
\text { lateral compartments injected } \\
\text { with AboBoNT-A }\end{array}$ & $\begin{array}{l}\text { Decreased intramuscular } \\
\text { pressure \& eliminated } \\
\text { exertional pain up to } 9 \\
\text { months }\end{array}$ & IV \\
\hline \multirow{10}{*}{$\begin{array}{l}\text { Functional Popliteal } \\
\text { Artery Entrapment } \\
\text { Syndrome (FPAES) }\end{array}$} & \multirow{3}{*}{ Murphy [15] } & \multirow{3}{*}{ Case Report } & $\begin{array}{l}\text { 50U medial gastrocnemius } \\
\text { heads bilaterally }\end{array}$ & $\begin{array}{l}1 \text { week decrease in strength } \\
\text { \& function }\end{array}$ & \multirow{3}{*}{$\mathrm{V}$} \\
\hline & & & \multirow{2}{*}{$\begin{array}{l}\text { Ceased lower body exercise } \\
\text { for } 1 \text { week \& gradual return to } \\
\text { training over } 2 \text { weeks }\end{array}$} & $\begin{array}{l}\text { Beneficial effect on pain } \\
\text { during sport }\end{array}$ & \\
\hline & & & & $\begin{array}{c}\text { 4-week decrease in peak } \\
\text { arterial velocity \& increase in } \\
\text { vessel size }\end{array}$ & \\
\hline & \multirow{4}{*}{ Hislop [16] } & \multirow{4}{*}{ Case Series } & \multirow{4}{*}{$\begin{array}{l}27 \text { patients injected at level or } \\
\text { artery occlusion }\end{array}$} & $0 \%$ no worse off & \multirow{4}{*}{ IV } \\
\hline & & & & $59 \%$ good response & \\
\hline & & & & $22 \%$ mixed & \\
\hline & & & & $19 \%$ no response & \\
\hline & \multirow{3}{*}{$\begin{array}{c}\text { Isner-Horobeti } \\
\text { [17] }\end{array}$} & \multirow{3}{*}{ Case report } & \multirow{3}{*}{$\begin{array}{l}400 \mathrm{U} \text { of AboBoNT-A in proximal } \\
\text { third of medial \& lateral } \\
\text { gastrocnemius bilaterally }\end{array}$} & No pain after 1 month & \multirow{3}{*}{$\mathrm{V}$} \\
\hline & & & & $\begin{array}{l}2.5 \text { months no compromise in } \\
\text { blood flow }\end{array}$ & \\
\hline & & & & $\begin{array}{l}4 \text { months - return to sport } \\
\text { without issues }\end{array}$ & \\
\hline \multirow{7}{*}{ Lateral Epicondylitis } & \multirow{3}{*}{ Creuze [19] } & \multirow{3}{*}{$\begin{array}{c}\text { Randomized, Double- } \\
\text { Blinded, Placebo- } \\
\text { Control }\end{array}$} & \multirow{3}{*}{$\begin{array}{c}60 \text { patients } 40 \mathrm{U} \text { AboBoNT-A vs } \\
\text { saline into ECRB }\end{array}$} & $\begin{array}{l}3 \text {-month follow up: } 51.7 \% \\
\text { reported }>50 \% \text { reduction in } \\
\text { initial pain intensity }\end{array}$ & \multirow{3}{*}{ I } \\
\hline & & & & $\begin{array}{l}\text { Pain intensity \& effect on } \\
\text { quality of life significantly } \\
\text { lower }\end{array}$ & \\
\hline & & & & $\begin{array}{l}\text { Transitory paresis of 3rd } \\
\text { finger on extension } 17.2 \% \text { in } \\
\text { BoNT-A group }\end{array}$ & \\
\hline & Lian [20] & $\begin{array}{l}\text { Systematic Review } \\
\text { \& Meta-analysis of } \\
\text { Randomized Placebo- } \\
\text { Controlled Trials }\end{array}$ & 3 studies, 237 patients for BoNT & $\begin{array}{l}\text { 5-26 weeks number needed } \\
\text { to treat (NNT) pain relief } 18 \\
\qquad(14-31)\end{array}$ & I \\
\hline & Dong [21] & $\begin{array}{c}\text { Systematic review } \\
\text { \& Bayesian network } \\
\text { meta-analysis }\end{array}$ & & $\begin{array}{l}\text { Botulinum toxin better than } \\
\text { placebo and corticosteroid } \\
\text { injection }\end{array}$ & I \\
\hline & Krogh [22] & $\begin{array}{l}\text { Systematic Review \& } \\
\text { Network Meta-analysis } \\
\text { of Randomized } \\
\text { Controlled Trials }\end{array}$ & 4 trials assessing BoNT & $\begin{array}{c}\text { Marginal pain benefit, caused } \\
\text { temporary paresis of finger } \\
\text { extension, all trials high risk } \\
\text { of bias }\end{array}$ & I \\
\hline & Oskarsson [23] & Case series & $\begin{array}{l}1 \mathrm{U} / \mathrm{kg} \text { AboBoNT-A injected } 2 \\
\text { fingerbreadths distal to lateral } \\
\text { epicondyle, upper dose limit of } \\
100 \mathrm{U} / \text { muscle }\end{array}$ & $\begin{array}{l}\text { Improves intramuscular } \\
\text { blood flow, aerobic } \\
\text { metabolism, functional } \\
\text { activity, and relieves pain at } \\
\text { 12-month follow up }\end{array}$ & IV \\
\hline $\begin{array}{l}\text { Accessory Soleus } \\
\text { Muscle }\end{array}$ & $\begin{array}{c}\text { Isner-Horobeti } \\
{[24]}\end{array}$ & Case Series & 280-1000U injected & $\begin{array}{c}\text { Disappearance of exertional } \\
\text { pain and resumption of } \\
\text { normal activity up to } 10 \text { years } \\
\text { after injection }\end{array}$ & IV \\
\hline
\end{tabular}




\begin{tabular}{|c|c|c|c|c|c|}
\hline Task-specific dystonia & Ahmad [26] & $\begin{array}{c}\text { Retrospective case } \\
\text { series }\end{array}$ & Runner's dystonia 13 patients & $7 / 9$ received benefit & IV \\
\hline & $\begin{array}{c}\text { Cutsforth- } \\
\text { Gregory [27] }\end{array}$ & Retrospective review & $\begin{array}{c}20 \text { patients (13 runners, } 7 \text { non- } \\
\text { runners) }\end{array}$ & Beneficial in 4/7 patients & III \\
\hline & Wu 2006 & Case series & Runner's dystonia, 5 cases & 2 patients benefited & IV \\
\hline
\end{tabular}

\section{Table $2 \mathrm{~b}$.}

\begin{tabular}{|c|c|c|c|c|c|}
\hline \multicolumn{6}{|c|}{ Muscular Imbalance Syndromes } \\
\hline Pathology & Author & Design & Protocol & Results & Evidence \\
\hline \multirow[t]{4}{*}{$\begin{array}{l}\text { Anterior Knee } \\
\quad \text { Pain }\end{array}$} & Stephen [30] & Case series & $\begin{array}{l}\text { Ultrasound -guided AboBoNT-A } \\
\text { injection into tensor fascia latae } \\
\text { with physical therapy }\end{array}$ & $\begin{array}{l}\text { Significant improvement in Anterior } \\
\text { Knee Pain Scale Score up to } 5 \text { years }\end{array}$ & IV \\
\hline & Singer [31] & Case series & $\begin{array}{l}\text { 500U AboBoNT-A in vastus } \\
\text { lateralis }\end{array}$ & $\begin{array}{l}\text { Improvement at } 12 \text { weeks based on } \\
\text { Anterior Knee Pain Scale Score }\end{array}$ & IV \\
\hline & \multirow[b]{2}{*}{ Cullen [32] } & \multirow[b]{2}{*}{ Review \& case series } & $\begin{array}{l}\text { 1) 500U AboBoNT-A to L upper } \\
\text { hamstrings }\end{array}$ & $\begin{array}{l}\text { 1) Isolated gluteal muscle } \\
\text { contraction in } 2 \text { weeks }\end{array}$ & \multirow[b]{2}{*}{ IV } \\
\hline & & & $\begin{array}{l}\text { 2) } 180 \mathrm{U} \text { in distal third of vastus } \\
\text { lateralis \& } 120 \mathrm{U} \text { to tensor fascia } \\
\text { latae with repeat of } 500 \text { into } \\
\text { each after } 5 \text { months }\end{array}$ & $\begin{array}{l}\text { 2) Improved pain \& activity } \\
\text { progression at } 2 \text { weeks with pain } \\
\text { after return to full weight bearing. } \\
\text { With second injection, improved } \\
\text { pain and able to return to play. }\end{array}$ & \\
\hline \multirow[t]{2}{*}{$\begin{array}{l}\text { Piriformis } \\
\text { Syndrome }\end{array}$} & Yoon [35] & $\begin{array}{l}\text { Prospective, case } \\
\text { control }\end{array}$ & $\begin{array}{l}\text { 150U AboBoNT-A injected by } \\
\text { CT-guidance into piriformis }\end{array}$ & $\begin{array}{l}\text { Significant reductions in pain at } \\
\text { week } 4,8 \text {, and } 12\end{array}$ & II \\
\hline & Lang [36] & Case series & $\begin{array}{l}\text { Piriformis injected once with } \\
5000 \mathrm{U} \text { of BoNT-B under EMG- } \\
\text { guidance }\end{array}$ & $\begin{array}{c}\text { Significant reductions in VAS for } \\
\text { buttocks } \& \text { hip pain at weeks } 4,12 \text {, } \\
\text { and } 16 \text { and for low back pain at } \\
\text { weeks } 2,12 \text {, and } 16\end{array}$ & IV \\
\hline \multirow[t]{2}{*}{$\begin{array}{l}\text { Rotator Cuff } \\
\text { Impingement }\end{array}$} & Lin [37] & $\begin{array}{c}\text { Systematic Review, } \\
\text { Pairwise \& Network } \\
\text { Meta-analysis of } \\
\text { Randomized Controlled } \\
\text { trials }\end{array}$ & & $\begin{array}{l}\text { Ineffective for rotator cuff } \\
\text { tendinopathy }\end{array}$ & I \\
\hline & Lee [38] & Case series & $\begin{array}{l}\text { 2500U BoNT-B subacromial } \\
\text { bursa vs triamcinolone }\end{array}$ & $\begin{array}{l}\text { At } 3 \text { months, BT decreased NRS and } \\
\text { DASH \& greater shoulder abduction } \\
\text { than triamcinolone }\end{array}$ & IV \\
\hline
\end{tabular}

Table 2c.

\begin{tabular}{|c|c|c|c|c|c|}
\hline \multicolumn{5}{|c|}{ Bioprotection } & Results \\
\hline Pathology & Author & Design & Protocol & Evidence \\
\hline Rotator cuff repair & Gilotra [39] & Rat model & $\begin{array}{c}\text { 84 supraspinatus tendons injected } \\
\text { w/ saline or examined at 2, 4, 8, } \\
\text { and 12 weeks }\end{array}$ & $\begin{array}{c}\text { May help protect repair but } \\
\text { cast immobilization plus BoNT } \\
\text { administration was harmful to healing }\end{array}$ & II \\
\hline Achilles tendon repair & Reuter [40] & Case series & $\begin{array}{c}\text { 200-300U in gastrocnemius \& } \\
\text { soleus }\end{array}$ & $\begin{array}{c}\text { 2 years improved pain and dorsiflexion } \\
\text { of 21 degrees reached }\end{array}$ & IV \\
\hline
\end{tabular}

\section{B Piriformis syndrome}

- Background: Piriformis syndrome is caused by prolonged or excessive contraction of the piriformis muscle associated with pain in the buttocks, hips, and lower limbs because of the close proximity to the sciatic nerve. BoNT-A reduces muscle hypertonia as well as muscle contracture and pain inhibiting substance $P$ release and other inflammatory factors [33,34]. The prospective study by Yoon [35] evaluated the efficacy of a single, low-dose injection of AboBoNT-A in 29 patients. Pain intensity scores were significantly lower at 4, 8, and 12 weeks after treatment than at baseline. Four weeks after treatment, physical functioning, bodily pain, general health, vitality and social functioning improved significantly from baseline. The prospective study by Lang [36] evaluated the clinical safety and efficacy of BoNT-B in reducing myofascial pain associated with piriformis syndrome in 20 patients. Significant reductions in mean visual analog scale scores for buttock and hip pain were noted at weeks 4,12, and 16 and for low back pain at weeks 2, 12, and 16. Visual analog scale scores for general and low back pain, pain radiating into lower limbs, and tingling were significantly lower at week 2 after injection, suggesting early onset.

- Conclusion - Grade B Recommendation: There is level II-IV evidence that BoNT can improve pain attributed to piriformis syndrome.

\section{Rotator cuff impingement}

- Background: Rotator cuff impingement occurs when the myotendinous junction of the supraspinatus tendon comes into contact with either the undersurface of the acromion, a subacromial 
spur or an enlarged acromioclavicular joint. BoNT has potential to alleviate shoulder impingement syndrome by decreasing muscle overactivity. Lin [37] published a pairwise and network meta-analysis of 18 studies to compare effectiveness of injection therapies in rotator cuff tendinopathy. The randomized prospective study by Lee [38] examined 61 patients treated with BoNT-B (BT group) or triamcinolone injection (TA group) under ultrasound guidance. Both groups obtained a significant improvement of Numeric Rating Scale, Disability of Arm, Shoulder, and Hand, and active shoulder abduction at 1 and 3 months follow-up. BT group showed significantly better outcomes in terms of reduction of NRS and DASH at 3 months than TA group. BT group showed strong trend toward the larger degree of active shoulder abduction than the TA group at 3 months follow-up, as well.

- $\quad$ Conclusion - grade $\mathrm{C}$ recommendation: There is level IV evidence that BoNT can improve pain in patients with rotator cuff external impingement.

\section{Bio protection}

\section{A Rotator cuff repair}

- Background: Increased postoperative activity is a risk factor for impaired rotator cuff repair. Various protocols of immobilization have been recommended but not substantiated to allow the rotator cuff to heal without jeopardizing long-term range of motion. Because of patient compliance issues and biological challenges, optimizing the postoperative healing environment remains difficult. As BoNT causes temporary muscle paralysis, it may have a role in facilitating postoperative immobilization. Gilotra [39] evaluated the effects of BoNT-A and cast immobilization on tendon healing in a rat model. Eighty-four supraspinatus tendons were surgically transected and repaired in 42 Sprague-Dawley rats. After repair, supraspinatus muscle was injected with saline or BoNT-A. Half the shoulders were cast-immobilized for the entire postoperative period; half were allowed free cage activity. Botox alone and cast immobilization alone exhibited increased ultimate load compared with at 4 weeks. No difference in ultimate load occurred between Botox-only and cast-only groups. At 12 weeks, the Botox plus cast immobilization group was significantly weakest. A trend was shown toward decreased healing zone cross-sectional areas in casted groups (Table 3).

- Conclusion-Grade B: There is level II evidence that supraspinatus Botox injection after rotator cuff repair can help protect the repair. However, prolonged immobilization plus Botox administration proved harmful to rotator cuff healing in a rat tendon model.

\section{B Achilles tendon repair}

- Background: Although outcome after surgical repair of complete Achilles tendon rupture is good, some patients have ongoing problems with dorsiflexion of the ankle joint. Reuter [40] analyzed a case series of 8 patients with reduced ankle dorsiflexion 5 months after surgical repair of complete Achilles tendon rupture. All patients received at least 3 cycles of injections with 200-300 units of BoNT-A into the gastrocnemius and soleus muscle.
Weakening of the triceps sure by BoNT allowed patients to perform the required exercises and to tolerate casting at night. All patients were able to tolerate plantigrade foot position 9 months after beginning of BoNT treatment. At final follow-up after 2 years, pain had significantly improved, and a mean dorsiflexion of 21 degrees was reached.

- Conclusion-Grade C recommendation: There is level IV evidence that the BoNT can improve restricted dorsiflexion in patients after Achilles tendon repair.

\section{Nociception}

\section{A Osteoarthritis}

- Background: BoNT has been suggested to have an antinociceptive effect when applied to painful joints by blocking neurotransmission from nerve terminals of nociceptive fibers. The randomized control study by Eleopra [41] AboBoNT-A injections (400 units into adductors) versus placebo $(1.6 \mathrm{~mL}$ saline) to improve hip range of motion, pain and quality of life in patients with hip osteoarthritis. At Week 4, the Harris Hip Score (HHS) and Visual Analog Score (VAS) were significantly improved compared to the placebo. No significant changes were observed in muscle strength and quality of life. The review by Hassan [42] evaluated 18 studies demonstrating limited evidence for BoNT-type A in knee osteoarthritis. The control group in several studies included did not have a control. Therefore, due to the possibility of placebo effect and bias could not be eliminated. The case series by Mahowald followed eleven patients for 12 months after intra-articular injections of BoNT-A for refractory joint pain due to osteoarthritis, rheumatoid arthritis and psoriatic arthritis. For the lower extremity joints (knee and ankle), the mean maximum decrease in joint pain was $55 \%$ and the $36 \%$ improvement in the Timed Stands Test was noted at 4 to 10 weeks after injection. For the upper extremity (shoulder), there was a $71 \%$ mean maximum reduction in pain severity and active range of motion increased $67 \%$ in flexion and $42 \%$ in abduction. Duration of pain relief ranged from 3 to 12 months [43].

\section{- Conclusion}

i. No Recommendation (hip): There is 1 study of level I evidence that BoNT may improve range of motion and pain in hip osteoarthritis after BoNT- A injection into the adductors. If there were studies examining hip intraarticular injections of BoNT-A injections, the outcomes may be similar to knee osteoarthritis.

ii. No Recommendation (knee): There is insufficient evidence BoNT-type A injections may help pain related to knee osteoarthritis. As there has been evidence of pain improvement after BoNT-A injections in the periarticular hip musculature, there may be avenues of pain improvement for patients with knee osteoarthritis by injecting BoNT-A injections into the periarticular knee musculature. The pain-relieving mechanism of action may be similar to those described in the muscular imbalances and overactivity sections.

\section{Whiplash}

- $\quad$ Background: Whiplash associated disorder is commonly linked to motor vehicle accidents and sports injuries. Cervical 
injury is attributed to rapid extension followed by neck flexion. The exact pathophysiology of whiplash is uncertain but is suspected to involve aberrant muscle spasms causing a wide range of pain symptoms. BoNT is a proposed treatment to relieve the aforementioned symptoms by muscle relaxation. Teasel [44] reviewed 3 randomized controlled trials and 1 case series on the effect of BoNT-A injections in the treatment of chronic whiplashassociated disorder (WAD). Patients who received BoNT injections achieved greater and longer-lasting pain relief as well as improved Table 3: Levels of Evidence. range of motion but not enough to achieve significance. The randomized controlled trial by Braker [45] followed 20 patients with cervical myofascial pain 2-48 weeks after a whiplash injury. The patients received either 200 units of BoNT-A or equal volume of saline injected equally into 4 trigger points. Although the BoNT-A group consistently made larger improvements, between-group differences were nonsignificant with the exception that a greater percentage of patients in the BoNT-A group achieved a $50 \%$ reduction in pain intensity at 24 weeks (Table 3).

\begin{tabular}{|c|c|c|c|c|}
\hline \multirow{2}{*}{ Evidence } & \multicolumn{4}{|c|}{ Types of Studies } \\
\hline & Therapeutic & Prognostic & Diagnostic & $\begin{array}{c}\text { Economic and Decision } \\
\text { Analysis }\end{array}$ \\
\hline & $\begin{array}{c}\text { Investigating the results of } \\
\text { treatment }\end{array}$ & $\begin{array}{l}\text { Investigating the effect of a } \\
\text { patient characteristics on } \\
\text { the outcome of disease }\end{array}$ & $\begin{array}{l}\text { Investigating a diagnostic } \\
\text { test }\end{array}$ & $\begin{array}{l}\text { Developing an economic } \\
\text { model or decision model }\end{array}$ \\
\hline Level I & $\begin{array}{l}\text { High-quality randomized } \\
\text { trial with statistically } \\
\text { significant difference but } \\
\text { narrow CIs }\end{array}$ & & & \\
\hline $\begin{array}{l}\text { Systematic review of level } \\
\text { I RCTs (and study results } \\
\text { were homogenous) }\end{array}$ & $\begin{array}{l}\text { High-quality prospective } \\
\text { study (all patients were } \\
\text { enrolled at the same point } \\
\text { in their disease with } \geq \\
80 \% \text { follow-up of enrolled } \\
\text { patients) Systematic review } \\
\text { of level I studies }\end{array}$ & $\begin{array}{l}\text { Testing of previously } \\
\text { developed diagnostic } \\
\text { criteria on consecutive } \\
\text { patients (with universally } \\
\text { applied reference "gold" } \\
\text { standard) Systematic } \\
\text { review of level II studies }\end{array}$ & $\begin{array}{c}\text { Sensible costs and } \\
\text { alternatives; values } \\
\text { obtained from many } \\
\text { studies; with multiway } \\
\text { sensitivity analyses } \\
\text { Systematic review of level I } \\
\text { studies }\end{array}$ & \\
\hline Level II & $\begin{array}{c}\text { Lesser-quality RCT (e.g., < } \\
\text { 80\% follow-up, no blinding, } \\
\text { or improper randomization) } \\
\text { Prospective comparative } \\
\text { study Systematic review } \\
\text { of level II studies or level I } \\
\text { studies with inconsistent } \\
\text { results }\end{array}$ & $\begin{array}{l}\text { Retrospective study } \\
\text { Untreated controls from } \\
\text { an RCT Lesser-quality } \\
\text { prospective study (e.g., } \\
\text { patients enrolled at } \\
\text { different points in their } \\
\text { disease or }<80 \% \text { follow-up) }\end{array}$ & $\begin{array}{l}\text { Development of diagnostic } \\
\text { criteria on consecutive } \\
\text { patients (with universally } \\
\text { applied reference "gold" } \\
\text { standard) Systematic } \\
\text { review of level II studies }\end{array}$ & $\begin{array}{c}\text { Sensible costs and } \\
\text { alternatives; values } \\
\text { obtained from limited } \\
\text { studies; with multiway } \\
\text { sensitivity review } \\
\text { Systematic review of level } \\
\text { II studies }\end{array}$ \\
\hline Level III & $\begin{array}{c}\text { Case-control study } \\
\text { Retrospective comparative } \\
\text { study Systematic review of } \\
\text { level III studies }\end{array}$ & Case-control study & $\begin{array}{l}\text { Study of nonconsecutive } \\
\text { patients; without } \\
\text { consistently applied } \\
\text { reference "gold" standard } \\
\text { Systematic review of level } \\
\text { III studies }\end{array}$ & $\begin{array}{c}\text { Analyses based on limited } \\
\text { alternatives and costs; poor } \\
\text { estimates Systematic review } \\
\text { of level II studies }\end{array}$ \\
\hline Level IV & Case series & Case series & $\begin{array}{c}\text { Case-control study } \\
\text { Poor reference standard }\end{array}$ & $\begin{array}{l}\text { Analyses with no sensitivity } \\
\text { analyses }\end{array}$ \\
\hline Level V & Expert Opinion & Expert Opinion & Expert Opinion & Expert Opinion \\
\hline
\end{tabular}

The randomized controlled trial by Padberg [46] followed 40 patients with chronic WAD who received either 100 units of BoNT-A or $2 \mathrm{~mL}$. After 12 weeks, no significant differences in pain intensity or cervical range of motion were found between the 2 groups. Juan published a case series [47] 31 patients with WAD for $\geq 3$ months refractory to conservative treatment. A dose of 50 to 75 units of BoNT-A was injected into each patient's tender superficial muscles. Patient were also provided with a home exercise program. $77.4 \%$ of patients responded positively to the treatment. Significant improvements were seen in terms of both pain intensity and cervical ROM. In the randomized controlled trial by Freund, 30 patients with WAD for $\geq 6$ months refractory to conservative treatment who received 100 units AboBoNT-A or $1 \mathrm{~mL}$ saline delivered to the 5 most tender cervical trigger points. At 4 weeks, patients in the treatment group had better cervical ROM, and less neck, head and shoulder pain than those in the placebo group.

- Conclusion - No Recommendation: There is currently insufficient evidence for the treatment of WAD with BoNT. However, there is evolving evidence that BoNT may be helpful for pain management, but studies thus far have not reached significance (Table 4). 
Table 4: Grades of Recommendation.

\begin{tabular}{|c|c|}
\hline Grade & Description \\
\hline A & Good evidence (Level-I studies with consistent findings) for or against recommending intervention \\
\hline B & Fair evidence (Level-II or III studies with consistent findings) for or against recommending intervention. \\
\hline C & $\begin{array}{c}\text { Conflicting or poor-quality evidence (Level-IV or V studies) not allowing a recommendation for or against } \\
\text { intervention. }\end{array}$ \\
\hline I & There is insufficient evidence to make a recommendation. \\
\hline
\end{tabular}

\section{Conclusion}

BoNT has emerging evidence of multiple beneficial applications in sports medicine. The evidence is strongest for plantar fasciitis and hip osteoarthritis. It is an effective and transient therapeutic option that may improve return to play times.

\section{References}

1. Jabbari B (2015) Botulinum Toxin Treatment of Pain Disorders. Springer, New York, USA.

2. Riddle DL, Schappert SM (2004) Volume of ambulatory care visits and patterns of care for patients diagnosed with plantar fasciitis: a national study of medical doctors. Foot Ankle Int 25(5): 303-310.

3. Riddle DL, Pulisic M, Pidcoe P, Johnson RE (2003) Risk factors for plantar fasciitis: a matched case-control study. J Bone Joint Surg Am 85(5): 872 877.

4. Lemont H, Ammirati KM, Usen N (2003) Plantar fasciitis: a degenerative process (fasciosis) without inflammation. J Am Podiatr Med Assoc 93(3): 234-237.

5. Schepsis AA, Leach RE, Gorzyca J (1991) Plantar fasciitis: Etiology, treatment, surgical results, and review of the literature. Clin Orthop Relat Res 266: 185-196.

6. Tsikopoulos K, Vasiliadis HS, Mavridis D (2016) Injection therapies for plantar fasciopathy ('plantar fasciitis'): a systematic review and network meta-analysis of 22 randomised controlled trials. Br J Sports Med 50(22): 1367-1375.

7. Peerbooms JC, Lodder P, den Oudsten BL, Doorgeest K, Schuller HM, et al. (2019) Positive Effect of Platelet-Rich Plasma on Pain in Plantar Fasciitis: A Double-Blind Multicenter Randomized Controlled Trial. Am J Sports Med 47(13): 3238-3246.

8. Shetty SH, Dhond A, Arora M, Deore S (2019) Platelet-Rich Plasma Has Better Long-Term Results Than Corticosteroids or Placebo for Chronic Plantar Fasciitis: Randomized Control Trial. J Foot Ankle Surg 58(1): 4246.

9. Elizondo-Rodriguez J, Araujo-Lopez Y, Moreno-Gonzalez JA, CardenasEstrada E, Mendoza-Lemus 0, et al. (2013) A comparison of botulinum toxin a and intralesional steroids for the treatment of plantar fasciitis: a randomized, double-blinded study. Foot Ankle Int 34(1): 8-14.

10. Babcock MS, Foster L, Pasquina P, Jabbari B (2005) Treatment of Pain Attributed to Plantar Fasciitis with Botulinum Toxin A: A Short-Term, Randomized, Placebo-Controlled, Double-Blind Study. Am J Phys Med Rehabil 84(9): 649-654.

11. Baria MR, Sellon JL (2016) Botulinum Toxin for Chronic Exertional Compartment Syndrome: A Case Report With 14 Month Follow-Up Clin J Sport Med 26(6): e111-e113.

12. Isner-Horobeti ME, Dufour SP, Blaes C, Lecocq J (2013) Intramuscular Pressure Before and After Botulinum Toxin in Chronic Exertional Compartment Syndrome of the Leg: A Preliminary Study. Am J Sports Med 41(11): 2558-2566.
13. Isner-Horobeti ME, Muff G, Masat J, Daussin JL, Dufour SP, et al. (2015) Botulinum Toxin as a Treatment for Functional Popliteal Artery Entrapment Syndrome. Med Sci Sports Exerc 47(6): 1124-1127.

14. Hislop M, Kennedy D, Cramp B, Dhupelia S (2014) Functional Popliteal Artery Entrapment Syndrome: Poorly Understood and Frequently Missed? A Review of Clinical Features, Appropriate Investigations, and Treatment Options. Journal of Sports Medicine pp: 1-8.

15. Murphy M, Charlesworth J, Koh E (2017) The effects of Botulinum Toxin injection in an elite sportsman with Functional Popliteal Artery Entrapment Syndrome: A case report. Phys Ther Sport 27: 7-11.

16. Hislop M, Brideaux A, Dhupelia S (2017) Functional popliteal artery entrapment syndrome: use of ultrasound guided Botox injection as a non-surgical treatment option. Skeletal Radiol 46(9): 1241-1248.

17. Oskarsson E, Piehl Aulin K, Gustafsson BE, Pettersson K (2009) Improved intramuscular blood flow and normalized metabolism in lateral epicondylitis after botulinum toxin treatment. Scand J Med Sci Sports 19(3): 323-328.

18. Creuzé A, Petit H, de Sèze M (2018) Short-Term Effect of Low-Dose, Electromyography-Guided Botulinum Toxin A Injection in the Treatment of Chronic Lateral Epicondylar Tendinopathy: A Randomized, DoubleBlinded Study. J Bone Joint Surg Am 100(10): 818-826.

19. Lian J, Mohamadi A, Chan JJ, Hanna P, Hemmati D, et al. (2019) Comparative Efficacy and Safety of Nonsurgical Treatment Options for Enthesopathy of the Extensor Carpi Radialis Brevis: A Systematic Review and Meta-analysis of Randomized Placebo-Controlled Trials. Am J Sports Med 47(12): 3019-3029.

20. Dong W, Goost H, Lin XB, Burger C, Paul C, et al. (2016) Injection therapies for lateral epicondylalgia: a systematic review and Bayesian network meta-analysis. Br J Sports Med 50(15): 900-908.

21. Krogh TP, Bartels EM, Ellingsen T, Stengaard PK, Buchbinder R, et al. (2013) Comparative Effectiveness of Injection Therapies in Lateral Epicondylitis: A Systematic Review and Network Meta-analysis of Randomized Controlled Trials. Am J Sports Med 41(6): 1435-1446.

22. Dhungana S, Jankovic J (2013) Yips and other movement disorders in golfers: Golfer's Yips: A Review. Mov Disord 28(5): 576-581.

23. Ahmad O, Ghosh P, Stanley C, Karp B, Hallett M, et al. (2018) Electromyographic and Joint Kinematic Patterns in Runner's Dystonia. Toxins 10(4): 166.

24. Cutsforth-Gregory JK, Ahlskog JE, McKeon A, Burnett MS, Matsumoto JY et al. (2016) Repetitive exercise dystonia: A difficult to treat hazard of runner and non-runner athletes. Parkinsonism Relat Disord 27: 74-80.

25. Wu LJ, Jankovic J (2006) Runner's dystonia. J Neurol Sci 251(1-2): 73-76.

26. Cullen DM, Boyle JJ, Silbert PL, Singer BJ, Singer KP (2007) Botulinum toxin injection to facilitate rehabilitation of muscle imbalance syndromes in sports medicine. Disabil Rehabil 29(23): 1832-1839.

27. Stephen JM, Urquhart DW, van Arkel RJ, Ball S, Jaggard MK, et al. (2016) The Use of Sonographically Guided Botulinum Toxin Type A (Dysport) Injections into the Tensor Fasciae Latae for the Treatment of Lateral Patellofemoral Overload Syndrome. Am J Sports Med 44(5): 1195-1202. 
28. Singer BJ, Silbert PL, Song S, Dunne JW, Singer KP (2011) Treatment of refractory anterior knee pain using botulinum toxin type A (Dysport) injection to the distal vastus lateralis muscle: a randomised placebo controlled crossover trial. Br J Sports Med 45(8): 640-645.

29. Silbert BI, Singer BJ, Silbert PL, Gibbons JT, Singer KP (2012) Enduring efficacy of Botulinum toxin type A injection for refractory anterior knee pain. Disabil Rehabil 34(1): 62-8.

30. Cass SP (2015) Piriformis Syndrome: A Cause of Nondiscogenic Sciatica. Curr Sports Med Rep 14(1): 41-44.

31. Santamato A, Micello M, Valeno G, Beatrice R, Cinone N, et al. (2015) Ultrasound-Guided Injection of Botulinum Toxin Type A for Piriformis Muscle Syndrome: A Case Report and Review of the Literature. Toxins 7(8): 3045-3056.

32. Yoon SJ, Ho J, Kang HY, Lee SH, Kim KI, et al. (2007) Low-Dose Botulinum Toxin Type A for the Treatment of Refractory Piriformis Syndrome. Pharmacotherapy 27(5): 657-665.

33. Lang AM (2004) Botulinum Toxin Type B in Piriformis Syndrome. American Journal of Physical Medicine \& Rehabilitation 83(3): 198-202.

34. Lin MT, Chiang CF, Wu CH, Huang YT, Tu YK, et al. (2019) Comparative Effectiveness of Injection Therapies in Rotator Cuff Tendinopathy: A Systematic Review, Pairwise and Network Meta-analysis of Randomized Controlled Trials. Arch Phys Med Rehabil 100(2): 336-349.

35. Lee JH, Lee SH, Song SH (2011) Clinical Effectiveness of Botulinum Toxin Type B in the Treatment of Subacromial Bursitis or Shoulder Impingement Syndrome. Clin J Pain 27(6): 523-528.

36. Gilotra MN, Shorofsky MJ, Stein JA, Murthi AM (2016) Healing of rotator cuff tendons using botulinum toxin A and immobilization in a rat model. BMC Musculoskelet Disord 17(1): 127.

37. Reuter I, Lorbach O, Mehnert S, Kaps M, Engelhardt M (2010) Botulinum toxin improves reduced dorsiflexion after Achilles tendon surgery. Knee Surg Sports Traumatol Arthrosc 18(2): 265-268.
38. Eleopra R, Rinaldo S, Lettieri C, Santamato A, Bortolotti P, et al. (2018) AbobotulinumtoxinA: A New Therapy for Hip Osteoarthritis. A Prospective Randomized Double-Blind Multicenter Study. Toxins 10(11): 448.

39. Hassan F, Murrell WD, Refalo A, Maffulli N (2018) Alternatives to Biologics in Management of Knee Osteoarthritis. Sports Med Arthrosc Rev 26(2): 79-85.

40. Mahowald ML1, Singh JA, Dykstra D (2006) Long term effects of intraarticular botulinum toxin A for refractory joint pain. Neurotox Res 9 (23): 179-88.

41. Teasell RW, McClure JA, Walton D, Pretty J, Salter K, et al. (2010) A Research Synthesis of Therapeutic Interventions for WhiplashAssociated Disorder (WAD): Part 5 - Surgical and Injection-Based Interventions for Chronic WAD. Pain Res Manag 15(5): 323-334.

42. Braker C, Yariv S, Adler R, Badarny S, Eisenberg E (2008) The analgesic effect of botulinum-toxin A on postwhiplash neck pain. Clin J Pain 24(1): 5-10.

43. Padberg M, de Bruijn SFTM, Tavy DLJ (2007) Neck pain in chronic whiplash syndrome treated with botulinum toxin. A double-blind, placebo-controlled clinical trial. J Neurol 254(3): 290-295.

44. Juan FJ (2004) Use of botulinum toxin-A for musculoskeletal pain in patients with whiplash associated disorders [ISRCTN68653575]. BMC Musculoskelet Disord 5(1): 5.

45. Freund BJ, Schwartz M (2002) Use of Botulinum Toxin in Chronic Whiplash-Associated Disorder. Clin J Pain 18(6): S163-168.

46. Okike K, Kocher M (2011) Chapter 14 Evidence-Based Orthopaedics: Levels of Evidence and Guidelines in Orthopaedic Surgery in Orthopaedic Surgery Knowledge Update 10. American Academy of Orthopaedic Surgeons pp: 157-165.

47. Wright JG, Einhorn TA, Heckman JD (2005) Grades of Recommendation. JBJS 87(9): 1909 -1910.

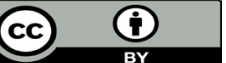

This work is licensed under Creative Commons Attribution 4.0 License

To Submit Your Article Click Here:

Submit Article

DOI: $10.32474 / O S M O A J .2020 .03 .000169$

$\begin{gathered}\text { Orthopedics and Sports Medicine } \\ \text { Open Access Journal }\end{gathered}$
Assets of Publishing with us
- Global archiving of articles
- Immediate, unrestricted online access
- Rigorous Peer Review Process
- Authors Retain Copyrights
- Unique DoI for all articles

